INPLASY

PROTOCOL

To cite: Liu et al. Virtual Reality in Improving Walking Ability and Balance Ability of

Parkinson's Patients: A Metaanalysis. Inplasy protocol 202090044. doi:

10.37766/inplasy2020.9.0044

Received: 10 September 2020

Published: 10 September 2020

Corresponding author:

Xiaoquan Zhang

473971576@qq.com

Author Affiliation:

Dalian University of

Technology

Support: No.

Review Stage at time of this submission: Data analysis.

Conflicts of interest: No.

\section{Virtual Reality in Improving Walking Ability and Balance Ability of Parkinson's Patients: A Meta-analysis}

Liu, Y1; Zhang, XQ².

Review question / Objective: To evaluate the effect of virtual reality (VR) on patients with Parkinson's disease (PD).

Condition being studied: VR has a positive effect on the balance and exercise ability of PD patients, but more randomized trials are needed to test its effect.

Information sources: Web of science, PubMed, CNKI, wanfang, weipu.

INPLASY registration number: This protocol was registered with the International Platform of Registered Systematic Review and Meta-Analysis Protocols (INPLASY) on 10 September 2020 and was last updated on 10 September 2020 (registration number INPLASY202090044).

\section{INTRODUCTION}

Review question / Objective: To evaluate the effect of virtual reality (VR) on patients with Parkinson's disease (PD).
Condition being studied: VR has a positive effect on the balance and exercise ability of PD patients, but more randomized trials are needed to test its effect. 


\section{METHODS}

Participant or population: Parkinson's disease.

Intervention: Virtual reality.

Comparator: A comparison between virtual reality and conventional rehabilitation.

Study designs to be included: Objective: To evaluate the effect of virtual reality (VR) on patients with Parkinson's disease (PD). Methods: the data of patients were retrieved from Web of science(WOS)、

PubMed、WeiPu、WanFang and CNKI in August 2020. Two researchers independently screened the literature, extracted the data and evaluated the quality of the literature, and then used RevMan5.3 for meta-analysis.

Eligibility criteria: Test for overall effect size: $Z(p<0.05)$.

Information sources: Web of science, PubMed, CNKI, wanfang, weipu.

Main outcome(s): Timed Up and Go test (TUG), Berg Balance Scale (BBS), 6-M walking test (6MWT), functional gait assessment (FGA) and dynamic gait index (DGI).

Strategy of data synthesis: (Parkinson OR "Parkinson's disease") AND (exergaming OR gaming OR "virtual reality gaming" OR "series gaming" OR"Wii" OR "virtual reality" OR VR OR"X box" OR Kinect OR "virtual reality therapy" OR"virtual reality exposure therapy" OR immersive OR illusion OR interactive OR augmented OR"computer*" OR"user-computer interface" OR simulation OR "exergam"” OR"exergaming or game or gaming" ) AND (gait OR balance OR equilibrium OR"functional reach" OR "postur" OR "dynamic postural control" OR "postural control" OR mobility OR ambulation OR walking).

Subgroup analysis: walking ability (about 6MWT, FGA, DGI).
Sensibility analysis: There are many options for VR intervention, as well as the control group.

Country(ies) involved: China.

Keywords: virtual reality, Parkinson, balance.

Contributions of each author:

Author 1 - Yang Liu.

Author 2 - Xiaoquan Zhang. 